the Convention is not legally binding on UK domestic legislation but places obligations on the government to ensure its laws are compliant. ${ }^{2}$ Complaints can be made to the UN commissioner where people with a disability feel that the Convention is not being appropriately implemented. It was not possible to determine whether any complaints had been received as a result of this definition.

In conclusion, the UK, in the sense of all three legislative areas, may receive a similar criticism to Spain from the UN Committee on the Rights of Persons with Disabilities when it reports, ${ }^{3}$ but it remains to be seen whether this will lead to widespread change in mental health legislation.

1 Kelly BD. An end to psychiatric detention? Implications of the United Nations Convention on the Rights of Persons with Disabilities. Br J Psychiatry 2014; 204: $174-5$.

2 Office for Disability Issues, HM Government. UK Initial Report on the UN Convention on the Rights of Persons with Disabilities. Office for Disability Issues, 2011.

3 Committee on the Rights of Persons with Disabilities. Concluding Observations of the Committee on the Rights of Persons with Disabilities: Spain. United Nations, 2011.

Daniel M. Bennett, Consultant Forensic Psychiatrist and Honorary Senior Lecture NHS Tayside and University of Aberdeen, Aberdeen, UK. Email: danielm.bennett@nhs.net

doi: 10.1192/bjp.205.1.76a

Author's reply: I agree with Szmukler that the 'fusion law' proposal would help shift detention criteria from the presence of mental disorder to the absence of decision-making capacity, and that a revised version of 'best interests' would be useful. In this context, it is interesting that the expert committee charged with advising the government on revising the Mental Health Act 1983 found that only a 'small minority' believed that 'a mental health act should authorise treatment in the absence of consent only for those who lack capacity' and 'if a person with a mental disorder who refused treatment was thought to pose a serious risk to others then he or she should be dealt with through the criminal justice system, not through a health provision.' There was, however, 'a much larger body of opinion which was prepared to accept the overriding of a capable refusal in a health provision on grounds of public safety in certain circumstances'. Notwithstanding this matter, I broadly agree with Szmukler that the 'fusion law' proposal would help move matters in the direction of greater compliance with the UN Convention on the Rights of Persons with Disabilities (CRPD).

Bennett's letter is also very constructive. His consideration of mental health legislation in Scotland and Northern Ireland clearly indicates that neither of those jurisdictions meets some of the apparent requirements of the CRPD, and provides further support for my conclusion that there is little evidence that the UK 'is ready for such profound change.' Ireland, incidentally, has recently made some progress towards greater compliance with the CRPD, with the publication of the Assisted Decision-Making (Capacity) Bill in 2013. ${ }^{3}$ There is, nonetheless, more work to be done in Ireland, as there is in England, Wales, Scotland, Northern Ireland and elsewhere, if the robust declarations of the CRPD are to generate meaningful and realistic protections for the full range of rights of people with mental illness.

1 Expert Committee. Review of the Mental Health Act 1983. Department of Health, 1999.

2 Kelly BD. An end to psychiatric detention? Implications of the United Nations Convention on the Rights of Persons with Disabilities. Br J Psychiatry 2014; 204; $174-5$
3 Kelly BD. The Assisted Decision-Making (Capacity) Bill 2013: content commentary, controversy. Ir J Med Sci 2014. Epub ahead of print (http:// dx.doi.org/10.1007/s11845-014-1096-1).

Brendan D. Kelly, Department of Adult Psychiatry, UCD School of Medicine and Medical Science, University College Dublin, Mater Misericordiae University Hospital, 62/63 Eccles Street, Dublin 7, Ireland. Email: brendankelly35@gmail.com

doi: 10.1192/bjp.205.1.77

\section{The significance of copy number variations in schizophrenia}

Rees et al ${ }^{1}$ seek to replicate the association with schizophrenia of copy number variants (CNVs) involving putative schizophrenia loci in a large case-control study. They conclude that 11 of the 15 previously implicated loci were strongly associated with schizophrenia. The odds ratios of these CNVs relative to schizophrenia range between around 2 and $>50$. The authors suggest that the findings now indicate a need for routine screening for CNVs.

However, I think there are grounds for reservations about the implication of these findings for the generality of cases of schizophrenia, both at the population level and in terms of public health initiatives. The authors report that one or more of the identified CNVs was present in $2.5 \%$ of the case group and in $0.9 \%$ of the control group. Let us assume that the prevalence of schizophrenia in the general population is around $0.5 \%$, as reported in the British National Psychiatric Morbidity Surveys. ${ }^{2-4}$ From this it is possible to calculate that, for every one person with schizophrenia who has one of these CNVs, there would be around 72 in the unaffected population. The positive predictive value (PPV) is the proportion of positive results of a test that are truly positive, and the PPV equivalent to these data can be calculated at $1.37 \%$ : in other words, this is the probability that someone with one of the identified CNVs has schizophrenia. If we change the assumed prevalence of schizophrenia to $1 \%$, the PPV rises to $2.73 \%$. The authors say: ' $\mathrm{g}$ ] iven their frequency, these findings therefore suggest that routine screening for CNVs should be made available and that the results will have immediate implications for genetic counselling, and given their comorbidity with other medical disorders, for patient management as well'. However, in my view, these values for PPVs make this conclusion questionable.

It is also of interest to use the authors' data to calculate the population attributable fraction (PAF): this is the notional amount by which the prevalence of an outcome would be reduced if the particular exposure were completely removed from the population. It reflects both the frequency of the given exposure and the strength of its effect. Using these data and, as before, assuming a prevalence of $0.5 \%$, the PAF is $0.618 \%$. If we assume a prevalence for schizophrenia of $1 \%$, this index changes very little, to $0.622 \%$. This is not a large value: we found a PAF of $14 \%$ for the link between psychosis and non-consensual sexual intercourse before the age of $16,{ }^{5}$ whereas a meta-analysis by Varese et $a l^{6}$ suggests that the PAF for all forms of childhood adversity in schizophrenia is 33\%.

The practical implications of CNVs in schizophrenia are thus in some doubt.

1 Rees E, Walters JTR, Georgieva L, Isles AR, Chambert KD, Richards AL, et al. Analysis of copy number variations at 15 schizophrenia-associated loci. $\mathrm{Br} J$ Psychiatry 2014; 204: 108-14.

2 Meltzer $\mathrm{H}$, Gill B, Petticrew M. The Prevalence of Psychiatric Morbidity among Adults Aged 16-64, Living in Private Households, in Great Britain. Office of Population Censuses and Surveys, Social Surveys Division, 1994.

3 Singleton N, Bumpstead R, O'Brien M. Psychiatric morbidity among adults living in private households, 2000. TSO (The Stationery Office), 2001.

4 McManus $\mathrm{S}$, Meltzer $\mathrm{H}$, Brugha TS, Bebbington PE, Jenkins R (eds) Adult Psychiatric Morbidity in England, 2007: Results of a Household Survey. NHS Information Centre for Health and Social Care, 2009. 
5 Bebbington $\mathrm{P}$, Jonas $\mathrm{S}$, Kuipers $\mathrm{E}$, King $\mathrm{M}$, Cooper $\mathrm{C}$, Brugha $\mathrm{T}$, et al. Childhood sexual abuse and psychosis: data from a cross-sectional nationa psychiatric survey in England. Br J Psychiatry 2011; 199: 29-37.

6 Varese F, Smeets F, Drukker M, Lieverse R, Lataster T, Viechtbauer W, et al. Childhood adversities increase the risk of psychosis: a meta-analysis of patient-control, prospective- and cross-sectional cohort studies. Schizophr Bull 2012; 38: 661-71.

Paul E. Bebbington, Emeritus Professor, Division of Psychiatry, University College London, London, UK. Email: p.bebbington@ucl.ac.uk

doi: 10.1192/bjp.205.1.77a

Authors' reply: We thank Professor Bebbington for his comments and for giving us the opportunity to clarify our recommendations relating to copy number variant $(\mathrm{CNV})$ testing in those with schizophrenia. We would like to make it clear that we were not suggesting universal screening of CNVs in healthy populations. Rather, we were suggesting that it is time to consider testing for CNVs in those with a diagnosis of schizophrenia. On reflection, we should have used the term 'genetic testing' rather than 'screening', and apologise for this ambiguity. In this sense the positive predictive value of CNVs for schizophrenia is irrelevant as the patient already has the disorder.

We believe that testing for pathogenic CNVs in schizophrenia should be considered for a number of reasons, but emphasise that this should only be undertaken with clear informed consent and in the context of professional genetic counselling. Among the potential benefits of knowing the carrier status of patients, physical health and informing patients about potential risks to family/offspring are the two areas that stand out.

Therefore, if we diagnose a patient with schizophrenia as a carrier of a pathogenic CNV, even though this will apply to only $2-3 \%$ of our patients, it could have important implications for their management. The identified CNVs can have an adverse impact on patients' health given that these CNVs are associated with obesity, epilepsy and cardiovascular disorders. This information could be crucial in guiding targeted monitoring and intervention, particularly given the increasing recognition of the effects of poor physical health and decreased life expectancy in schizophrenia. These factors may also be important considerations in the selection of the most appropriate medication.

Further, although the frequency of the implicated CNVs is low in schizophrenia, each of the 11 implicated CNVs can lead to a range of other disorders such as developmental delay, intellectual deficit, autism spectrum disorders, and a number of congenital anomalies. We have estimated that carriers of these CNVs have substantial risk of developing one of these serious disorders. ${ }^{1}$ The risk ranges from $10.6 \%$ for the duplication at $16 \mathrm{p} 13.11$ to nearly $100 \%$ for the deletion at 22q11.2, with an average of $42.8 \%$. The penetrance solely for schizophrenia is indeed relatively low, ranging from 2 to $12 \%$ (assuming a $1 \%$ lifetime risk for schizophrenia). Taken together, we feel that this information could be helpful to patients in making decisions about having children and potentially for their wider family.

There is currently a lack of research into the possible benefits and risks of such genetic testing and we would strongly advocate for such research before the implementation of CNV testing programmes. This should be informed by the wealth of experience in genetic counselling that has developed in other genetic disorders. We feel many patients and families would find this information helpful in rationalising a cause for the illness and that this may help lessen the guilt experienced by many families. We appreciate the chance to have begun this debate and would stress that the views of patients with schizophrenia and their families relating to genetic testing should be central to the debate and future research.
1 Kirov G, Rees E, Walters JT, Escott-Price V, Georgieva L, Richards AL, et al. The penetrance of copy number variations for schizophrenia and developmental delay. Biol Psychiatry 2014; 75: 378-85.

Elliott Rees, MRes, James T. R. Walters, PhD, MRCPsych, Michael J. Owen, PhD, FRCPsych, George Kirov, PhD, MRCPsych, Centre for Neuropsychiatric Genetics and Genomics, Institute of Psychological Medicine and Clinical Neurosciences, Cardiff University, Cardiff, UK. Email: kirov@cardiff.ac.uk

doi: 10.1192/bjp.205.1.78

\section{Getting real about risk}

The recent meta-analysis by Singh et $a l^{1}$ examined the proportion of violent people among those classified as high risk, known as the positive predictive value (PPV). They found that PPV is highly variable between studies and is most strongly associated with the base rate of violence in the whole risk-assessed group. They conclude that risk assessment is not a reliable indicator of absolute risk. We agree. The increased focus on the PPV of high-risk categories is a welcome development because it leads to a consideration of the number of people who might need to be assessed as high-risk for every true positive (actually violent) person. The number needed to assess is often a more relevant measure than those derived from the receiver operator curve and it clearly illustrates the lack of meaning in recent debates about the extent to which group data apply to individuals ${ }^{2}$ and the margins of error in particular risk predictions. ${ }^{3}$ However, we believe that the debate about risk assessment now needs to move beyond abstract notions relating solely to probability. A probability after all is simply a number between 0 and 1 , a number that is uninformative unless it is a probability of something specific.

Although not cited in Singh et al, we systematically examined PPV of risk categorisation after making generous assumptions about the statistical power of risk assessment. ${ }^{4}$ Unlike Singh et al, our paper focused on the main factor that actually determines base rates and thus PPV - the definition of violence according to violence severity. For example, using a risk assessment instrument with a sensitivity and specificity of $80 \%$ for the detection of different outcomes, the PPV for criminally violent behaviour over a year by people with schizophrenia might be about $4 \%$ under optimal conditions, whereas the same figure for homicide would be $0.04 \%$.

In the primary risk research, including that used by Singh et $a l,{ }^{1}$ a wide spectrum of violent events is amalgamated into an omnibus 'violent' category. These events range from common assault all the way to homicide. Each of these diverse events has different base rates and consequences, with more severe violence having lower base rate but leading to greater losses.

Risk assessment in mental health should start to consider the dimension of resulting loss. In areas outside mental health, risk is not a probability but is a quantum of loss - that is why we pay our insurance premium in money, yet have little idea of the likelihood of the loss of our possessions. In our view any study that does not consider the magnitude of resulting loss should not really be thought of as a 'risk assessment' and more properly should be referred to as 'probability assessment'. Although quantification of loss poses significant challenges, considering a definition of risk that includes the loss component re-emphasises two complex, important and unanswered questions. First, what actual psychiatric interventions in terms of cost/side-effects/benefits are indicated for those who are regarded as high risk, and yet should be withheld from patients classified as low risk? If the harm we consider is not severe, no costly, restrictive or intrusive treatment can be justified. If the harm considered is severe, it will also be rare. Therefore, what costly and intrusive intervention can be justified for the tiny proportion of false positives, or if the intervention is not costly or intrusive, why withhold it from 